\title{
Effect of Comprehensive Nursing Intervention on Intestinal Preparation Quality of Hospitalized Patients undergoing Colonoscopy
}

\author{
Li Liu, Fang Fang, Yingfeng Huang* \\ Department of Gastroenterology, The First Affiliated Hospital of Jinan University, Guangzhou 510660, China \\ *Corresponding author: Yingfeng Huang, 260244978@qq.com
}

\begin{abstract}
Objective: To explore the impact of comprehensive nursing intervention on the quality of intestinal preparation in the process of intestinal preparation for hospitalized patients undergoing colonoscopy. Methods: A total of 320 patients underwent colonoscopy in our department from January to June 2021. They were grouped by random number table. There were 160 cases in the control group and 160 cases in the observation group. The adverse reactions of intestinal preparation and the cleanliness of intestinal preparation were analyzed. Results: Through comprehensive nursing intervention, it can be found that the incidence of adverse reactions in the preparation of colonoscopy in the control group was $24.38 \%$, and that in the observation group was $13.48 \%$. The results showed that the incidence of adverse reactions in the preparation of colonoscopy was significantly lower than that in the control group $(\mathrm{P}<0.05)$, which indicates that the difference was statistically significant. In the control group, 105 people qualified in intestinal cleanliness score ( $>5$ points) during enteroscopy preparation, and the cleanliness qualification rate was $65.63 \%$. In the observation group, 139 people qualified in intestinal cleanliness score ( $>5$ points) during enteroscopy preparation, and the cleanliness qualification rate was $86.88 \%$. The cleanliness qualification rate of enteroscopy preparation was significantly higher than that of the control group, and $\mathrm{P}<$ 0.05 , which indicates that the difference was statistically significant. Conclusion: Comprehensive nursing intervention can promote the quality of preparation of patients for colonoscopy, improve the compliance of patients and improve the accuracy of examination effect.
\end{abstract}

Keywords: Comprehensive nursing intervention; Enteroscopy; Cleanliness

Publication date: November 2021; Online publication: November 30, 2021

\section{Introduction}

Early intervention can better control the disease, but most colorectal cancer patients are asymptomatic or mild in the early stage, resulting in missing the best treatment period ${ }^{[1]}$. At present, clinical diagnosis and screening mainly rely on colonoscopy, and the accuracy of the results is closely related to qualified intestinal preparation ${ }^{[2]}$. Previous studies showed that about $20 \%$ of patients affected the examination results due to unqualified intestinal preparation ${ }^{[3]}$. Comprehensive nursing intervention carries out effective evaluation according to the situation of patients, and formulates a practical and effective implementation plan of comprehensive nursing intervention to improve the quality of intestinal preparation of patients. Studies have shown that effective nursing intervention can improve the success rate of intestinal preparation and examination ${ }^{[4]}$. This paper studies the quality of intestinal preparation in hospitalized patients undergoing colonoscopy through comprehensive nursing intervention. 


\section{Materials and methods}

\subsection{General information}

A total of 320 patients underwent colonoscopy in our department from January to June 2021. They were grouped by random number table. There were 160 cases in the control group, including 78 male patients and 82 female patients, and 160 cases in the observation group, including 89 male patients and 71 female patients, which were generally not statistically significant and did not affect the results of the study.

\subsection{Methods}

(1) The control group was given routine nursing. Health education was conducted for precautions and preparation knowledge before the examination. At 18:00 on the day before the examination, the patient was instructed to take orally 1 bag of Compound Polyethylene Glycol Electrolyte Powders (II) (Heshuang, 68.56g/bag), dissolved in $1000 \mathrm{ml}$ water, and take $250 \mathrm{ml}$ every 15 minutes within 1 hour. The patient was instructed to take 2 bags of Heshuang orally at 6:00pm on the day, dissolved in 2000 $\mathrm{ml}$ water, and drank $250 \mathrm{ml}$ every 15 minutes within 2 hours. At the same time, the psychological status of patients was evaluated and targeted psychological intervention was given.

(2) The patients in the observation group were given comprehensive nursing intervention, including:

a) Health education: on the day of appointment, the responsible nurse shall introduce the purpose, scheme and relevant precautions of this study to the patients and their families, and the study can be carried out only after obtaining their informed consent. The responsible nurse will make a paper brochure with pictures and texts on the precautions during intestinal preparation, adverse reactions of intestinal preparation, pictures of Intestinal cleansing agent, usage and pictures of ideal defecation characteristics, which will be distributed to the patients on the day of appointment.

b) Intestinal preparation: patients were instructed to take Heshuang before examination. The usage and dosage were the same as those in the control group.

c) Defecation assessment: Nurses explain the process from taking medicine to the completion of intestinal cleaning to patients through pictures, from soft stool - paste stool - water sample stool, from dark yellow to light yellow, $6 \sim 8$ times, emphasizing that the completion of cleaning is based on the discharge of water sample stool.

d) Psychological intervention: One day before the examination, patients' doubts can be patiently answered through face-to-face communication, so as to improve patients' awareness of enteroscopy knowledge, so as to alleviate negative emotions such as fear; Before examination, yoga and meditation can be used to calm the patient's anxiety, tension and other negative emotions.

\subsection{Observation indexes}

Adverse reactions are classified as excellent, good, medium and poor. Excellent indicates that the patient has no symptoms such as vomiting, nausea and abdominal distention, good indicates that the patient has slight nausea and cold feeling, medium indicates that the patient has obvious adverse symptoms such as nausea, abdominal pain and headache, and poor indicates that the patient has serious adverse reactions such as shock and intestinal perforation. The sum of good, medium and poor is the total number of adverse reactions. The intestinal cleanliness was evaluated by the Boston intestinal score scale widely used in the world, which was completed by the doctors of the endoscopy center who were trained and qualified.

\subsection{Statistical methods}

The data of this study were processed by SPSS 20.0 statistical software. The measurement data is expressed by mean \pm standard deviation $(\bar{x} \pm s)$, and t-test is performed. The counting data is expressed by rate $(\%)$, and the line is $\chi^{2}$ inspection; If the test results show that $\mathrm{P}<0.05$, the difference is statistically significant. 


\section{Results}

\subsection{Incidence of adverse reactions during intestinal preparation in the two groups}

Through comprehensive nursing intervention, it can be found that during colonoscopy preparation in the control group, the number of excellent, good, medium and poor ratings were 121, 8, 10, 21 and 39 respectively, and the total number of adverse reactions ( $=$ good + medium + poor) was 39 , and the incidence of adverse reactions was $24.38 \%$ (Table 1). During colonoscopy preparation in the observation group, the number of excellent, good, medium and poor ratings were 141, 8, 6 and 5 respectively, and the total number of adverse reactions (= good + medium + poor) was 19 , and the incidence of adverse reactions was $13.48 \%$. The results showed that the incidence of adverse reactions in enteroscopy preparation was significantly lower than that in the control group, and $\mathrm{P}<0.05$ indicated that the difference was statistically significant.

Table 1. Comparison of the incidence of adverse reactions during intestinal preparation

\begin{tabular}{lcccc}
\hline Group & Control group $(\mathbf{n}=\mathbf{1 6 0})$ & Observation group $(\mathbf{n}=\mathbf{1 6 0})$ & $\chi^{\mathbf{2}}$ & P \\
\hline Excellent & 121 & 141 & & \\
Good & 8 & 8 & & \\
Middle & 10 & 6 & & \\
Poor & 21 & 5 & & \\
Adverse reaction & 39 & 19 & & \\
Adverse reaction rate $(\%)$ & 24.38 & 13.48 & 8.4233 & 0.0037 \\
\hline
\end{tabular}

\subsection{Comparison of qualified rate of intestinal cleanliness between the two groups}

Through comprehensive nursing intervention, it can be found that in the control group, the number of intestinal cleanliness scores of 9 points, 8 points, 7 points, 6 points and 1-5 points were 21, 23, 25, 36 and 55 respectively, the number of qualified ( $>5$ points) was 105 , and the qualified rate of cleanliness was $65.63 \%$ (Table 2). In the observation group, the number of intestinal cleanliness scores of 9 points, 8 points, 7 points, 6 points and $1-5$ points was 40,30,23, 46 and 21 respectively, the number of qualified (> 5 points) was 139 , and the qualified rate of cleanliness was $86.88 \%$. The cleanliness qualified rate of enteroscopy preparation observed was significantly higher than that of the control group, and $\mathrm{P}<0.05$ indicated that the difference was statistically significant.

Table 2. Comparison of qualified rate of intestinal cleanliness between the two groups

\begin{tabular}{lcccc}
\hline Group & Control group $(\mathbf{n}=\mathbf{1 6 0})$ & Observation group $(\mathbf{n}=\mathbf{1 6 0})$ & $\chi^{\mathbf{2}}$ & P \\
\hline 9 points & 21 & 40 & & \\
8 points & 23 & 30 & \\
7 points & 25 & 23 & \\
6 points & 36 & 46 & & \\
$1-5$ points & 55 & 21 & & \\
Cleanliness qualified (> 5 points) & 105 & 139 & 19.9482 & 0.0000 \\
Cleanliness qualified rate (\%) & 65.63 & 86.88 & & \\
\hline
\end{tabular}

\section{Discussion}

Colonoscopy is a common diagnosis and treatment measure for intestinal diseases. A clear field of vision is an important condition to ensure the smooth examination of doctors. Therefore, intestinal preparation must be carried out before operation. However, research shows that some patients are affected by diseases 
and lack of basic knowledge of colonoscopy, which affects the intestinal cleaning effect, making it difficult for them to tolerate colonoscopy psychologically and physiologically ${ }^{[5-6]}$.

To sum up, comprehensive nursing intervention can promote the quality of preparation of patients for colonoscopy, improve the compliance of patients and improve the accuracy of examination effect.

\section{Disclosure statement}

The authors declare no conflict of interest.

\section{References}

[1] Huang JJ, Su J, Zhou Y, et al., 2020, Using Medical Failure Mode and Effect Analysis to Improve the Quality of Intestinal Preparation Before Colonoscopy. Nursing of Integrated Traditional Chinese and Western Medicine (Chinese and English), 6(02): 8-12.

[2] Zhang XX, 2020, Effects of Different Doses of Polyethylene Glycol Electrolyte Combined with Defoamer on Intestinal Preparation Quality before Colonoscopy. China Medical University.

[3] Wang XX, et al., 2019, Effect of Intensive Education on Intestinal Cleanliness in Elderly Patients Undergoing Colonoscopy. Bachu Medical Journal, 2(04): 96-99+ 124.

[4] Ding XJ, Tang CL, et al., 2019, Effects of FOCUS-PDCA Model on Intestinal Preparation Quality Before Colonoscopy. Nnrsing Practice and Research, 16(22): 75-77.

[5] Guo R, Zhang J, Zhai HH, et al., 2019, Effects of Different Education Modes on Intestinal Cleansing before Colonoscopy. Chinese Journal of Medicine, 54(22): 1197-1200.

[6] Xu SL, Tang T, Chen J, et al., 2019, Analysis of Intestinal Cleansing Effect and Its Influencing Factors in Colonoscopy. Chinese Journal of Practical Nursing, (29): 2256-2262. 